COMMENT. The authors describe an encephalopathy associated with a prolonged febrile seizure and generally resulting in a poor outcome. In 10 of 17 patients, the infectious agent was isolated from the throat, but neither viral PCR analysis of the CSF nor cytokine levels were recorded. The encephalopathy was similar to that described with influenza A (Kawada J-I et al. J Infect Dis 2003;188:690-698; and HHV-6-associated febrile seizures (Akasaka M et al. Brain Dev 2005;27:30-33). Some viruses (eg influenza A, HHV-6) particularly prone to cause encephalopathy or complex febrile seizures result in excessive systemic immune and cytokine reponses (Millichap JG, Millichap JJ. J Infect Dis 2004;189:564-565). In febrile seizure patients CSF viral invasion is unusual, and the height of the fever has a more essential role in the febrile seizure mechanism than a specific viral neurotropism. Influenza-associated febrile seizure patients have significantly higher serum levels of pro-inflammatory cytokines than febrile patients without seizures (Masuyama $\mathrm{T}$ et al. Pediatr Neurol 2002;27:289-292). Cytokine levels are useful indicators of the severity of the infection and the associated encephalopathy (Ichiyama T et al. Cytokine 2004;27:31-37), and may help to distinguish the complex febrile seizure from an encephalopathy. Future research concerning the mechanism of encephalopathy complicating prolonged febrile seizures should include CSF viral PCR analysis, cytokine levels, and immune responses.

\title{
STATUS EPILEPTICUS INDUCED BY ANTIEPILEPTIC DRUGS
}

Adolescent and adult patients with idiopathic generalized epilepsy (IGE) who developed paradoxical video-EEG documented status epilepticus (SE) precipitated by inappropriate antiepileptic drugs (AEDs) were studied retrospectively at Hopital Pasteur, Nice; Hopital Rangueil, Toulouse; and Hopital Henri-Gastaut, Marseilles, France. Among 14 patients, aged 15-46 years, identified, 5 had typical absence SE (ASE), 5 had atypical ASE, 3 had atypical myoclonic SE (MSE), and one had typical MSE. All experienced an increase in seizures or new seizure types before occurrence of SE. Epilepsy had been misclassified as cryptogenic partial in 8 cases and cryptogenic generalized in 4 , leading to inappropriate choice of AEDs. All had received carbamazepine (CBZ) that caused seizure aggravation before referral. Polytherapy with phenytoin (PHT), vigabatrin (VGB) or gabapentin (GBP) had been used in 7 patients. Seizures were precipitated following dose increases of CBZ or of CBZ and PHT; by initiation of CBZ, VGB or GBP; and by a decrease of phenobarbital. Withdrawal of the inappropriate medication and substitution of valproate (VPA) monotherapy in 5 and polytherapy including VPA in 8 resulted in complete seizure control. Interictal EEGs after successful seizure control were normal or showed only rare bursts of spike-and-wave on a normal background. The correct diagnosis was juvenile absence epilepsy in 6 patients, juvenile myoclonic epilepsy in 4, epilepsy with grand mal on awakening in 2, and childhood absence epilepsy in 2. (Thomas P, Valton L, Genton P. Absence and myoclonic status epilepticus precipitated by antiepileptic drugs in idiopathic generalized epilepsy. Brain May 2006;129:1281-1292). (Respond: Pierre Thomas MD PhD, Unite Fonctionnelle EEG-Epileptologie, Service de Neurologie, Hopital Pasteur, 30 Voie Romaine, 06002 Nice, France).

COMMENT. Paradoxical exacerbation of IGE may result from inappropriate AEDs and may be expressed as absence or myoclonic status epilepticus. CBZ is most often implicated and is contraindicated in absence and juvenile myoclonic epilepsies. 